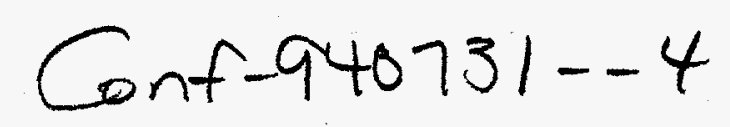

PNL-SA-24441

\title{
SIMULATING THE PRODUCTION OF FREE DEFECTS IN IRRADIATED METALS
}

\section{DISCLAIMER}

H. L. Heinisch

July 1994

This report was prepared as an account of work sponsored by an agency of the United States Government. Neither the United States Government nor any agency thereof, nor any of their employees, makes any warranty, express or implied, or assumes any legal liability or responsibility for the accuracy, completeness, or usefulness of any information, apparatus, product, or process disclosed, or represents that its use would not infringe privately owned rights. Reference herein to any specific commercial product, process, or service by trade name, trademark, manufacturer, or otherwise does not necessarily constitute or imply its endorsement, recommendation, or favoring by the United States Government or any agency thereof. The views and opinions of authors expressed herein do not necessarily state or reflect those of the United States Government or any agency thereof.

Presented at the

Second International Conference on Computer Simulation of

Radiation Effects in Solids

July 25-29, 1994

Santa Barbara, California

Prepared for

the U.S. Department of Energy

under Contract DE-AC06-76RLO 1830

Pacific Northwest Laboratory

Richland, Washington 99352 


\section{DISCLAIMER}

Portions of this document may be illegible in electronic image products. Images are produced from the best available original document. 


\author{
H. L. Heinisch \\ Pacific Northwest Laboratory ${ }^{*}$ \\ Richland, WA 99352, USA
}

\begin{abstract}
Under cascade-producing irradiation by high energy neutrons or charged particles, only a small fraction of the initially displaced atoms contribute to the population of free defects, i.e. those that are available to migrate throughout the metal and cause microstructural changes. Although, in principle, computer simulations of free defect production could best be done using molecular dynamics, in practice, the wide ranges of time and distance scales involved can be done only by a combination of atomistic models that employ various levels of approximation. An atomic-scale, multimodel approach has been developed that combines molecular dynamics, binary collision models and stochastic annealing simulation. The annealing simulation is utilized in calibrating binary collision simulations to the results of molecular dynamics calculations, as well as to model the subsequent migration of the defects on more macroscopic time and size scales. The annealing simulation and the method of calibrating the multimodel approach are discussed, and the results of simulations of cascades in copper are presented. The temperature dependence of free defect production following simulated annealing of isolated cascades in copper shows a differential in the fractions of free vacancies and interstitial defects escaping from the cascade above Stage $V$. This differential, a consequence of the direct formation of interstitial clusters in cascades and the relative thermal stability of vacancy and interstitial clusters during subsequent annealing, is the basis for the production bias mechanism of void swelling.
\end{abstract}

* Operated for the U.S. Department of Energy by Battelle Memorial Institute under Contract DE-AC06-76RLO 1830. 


\section{INTRODUCTION}

When cascade-producing radiation damage occurs in a solid, a distribution of single point defects and clustered point defects is produced in the local region where displacement damage is deposited. This defect distribution evolves in time. Some of the mobile defects interact within the cascade region, while others escape the cascade and interact globally as part of the "free" or "available" defects that are responsible for many radiation-induced microstructural changes. Only a small fraction of all the atoms initially displaced in a single cascade survive in the form of point defects, and, depending on the temperature, only a fraction of the survivors interact outside the cascade region.

There are four stages in the process of defect production in a cascade: 1.) the initial displacing collisions, 2.) formation of a thermal spike, 3.) quenching of the thermal spike, and 4.) annealing within the cascade. In the collisional phase, which lasts a few tenths of a picosecond, a primary recoil atom initiates a cascade of displacive collisions that continues until no atom contains enough energy to create further displacements. During the thermal spike stage, a molten zone is created as the collisional energy is shared among the atoms in a localized region having a high density of deposited energy. The identity of defects within the molten zone is largely lost, but the defects outside the molten zone (displaced atoms at the ends of replacement collision sequences, those ejected ballistically, and some vacancies) retain their identity. The displaced atoms become selfinterstitial atoms (SIA). Molecular dynamics (MD) simulations indicate that 
elastic interactions lead to clustering of SIAs outside the molten zone [1]. During the quenching stage, which lasts for about $10 \mathrm{ps,} \mathrm{the} \mathrm{molten} \mathrm{zone}$ returns to a condensed state containing a distribution of vacancies within it. During the annealing stage, which may last for seconds, further rearrangement and interaction of the remaining defects takes place by normal, thermallyactivated diffusion of mobile defects. It is in this stage that some of the mobile defects escape the cascade region to become the "free defects."

The four stages of cascade development occur over time scales differing by 15 orders of magnitude and can involve volumes of material containing millions of atoms. Hence, MD cannot be used to describe the entire cascade evolution. In this paper a methodology is discussed for simulating defect production through the annealing stage, well beyond the capabilities of MD, that incorporates information about the spatial distribution of individual defects. This methodology utilizes a discrete, defect-scale, stochastic annealing simulation code, ALSOME. Two examples of using ALSOME to extend our knowledge of the cascade damage state beyond the spatial and time constraints of MD calculations are presented here. First, ALSOME is used to describe the subsequent annealing stage of cascades in copper that have been simulated through the quenching stage by MD. The results illustrate for isolated cascades the key concept of the "production bias" considered in theories of void swelling [2]. In the second example, the role of annealing simulations in the calibration procedure for dealing with high energy cascades (those intractable by MD) by the multimodel approach (MMA) [3] is discussed and illustrated for cascades in copper. 


\section{The ALSOME Model}

In the stochastic annealing code ALSOME defects are the interacting entities. Each defect or defect cluster is associated with a lattice site. Mobile defects move by single, uncorrelated, random jumps to adjacent lattice sites. The next defect to jump is selected randomly from a distribution of the existing mobile defects that is weighted by the relative jump probabilities. Two defects coming within a defect-specific critical reaction distance are assumed to coalesce (cluster or annihilate), with the product defect (if any) residing at the target defect's lattice site. Thermally unstable defect clusters can dissociate one point defect at a time according to the relative probability of defect emission from the cluster.

The relative jump probabilities are calculated from the equation

$$
P(k, n, T)=P_{0} \exp \left(-E_{a}(k, n) / k T\right),
$$

where $k$ is the defect type (vacancy or SIA), $n$ is the cluster size, $T$ is the absolute temperature, and $E_{a}$ is the activation energy for the event. The preexponential factor $P_{0}$ is assumed constant (i.e., it has small variations relative to the differences in the exponential term). In the present calculations all probabilities are calculated relative to the jump probability of a single SIA at $300 \mathrm{~K}$. For conversion to real time, the jump frequency of a single interstitial is taken as $10^{13} \mathrm{~s}^{-1}$. 
In earlier work, summarized in Ref. 4, ALSOME was applied to the annealing of cascades through Stage III, to about room temperature in copper. Simulations were carried out to the point where no mobile defects remained in the cascade region. Therefore, the specific values used for the relative jump probabilities were not very important. It was found that, as long as the relative jump probabilities of the mobile species were ranked in the proper order (i.e., SIAs move well before vacancies), their actual values did not significantly affect the final results.

In the present work, where both dissociation and migration must be considered, and the actual annealing times and temperature dependence are important, it is necessary to use the best values for the relative probabilities of occurrence of all phenomena. Few of the activation energies have been determined experimentally, so for the present work we used calculated values determined by static MD calculations found in the literature, many of which were obtained with reliable long-range potentials derived from first principles. The static defect calculations indicate that vacancy clusters up to size $n=4$ and SIA clusters up to size $n=3$ have significant mobilities relative to single vacancies and SIAs, respectively. In addition, there is evidence from MD simulations [5] that small glissile interstitial loops readily form in cascades in copper, and these will glide in one dimension along <111> directions. The migration energy of these loops is estimated to be about equal to that of single SIAs [6]. 
The activation energies were tested in ALSOME by simulating the isochronal annealing of copper after electron irradiation at $4.2 \mathrm{~K}$ and comparing with resistivity recovery experiments [7]. Some adjustments and judgements regarding different calculated values were made as a result of these tests. A thorough review of calculations and experiments on defect migration and stability, with recommended values for activation energies, is necessary. The values of activation energies used in the present simulations are listed in Ref. 7.

\section{Annealing Simulations of MD Cascades}

Two $25 \mathrm{keV}$ cascades generated with MD were annealed using ALSOME. The cascades were produced by Diaz de la Rubia and Guinan [8] using the MOLDYCASK MD code, and they are the highest energy cascades generated by MD computer simulation to date. Their simulations followed the cascades for about the first 10 ps of their development, by which time the temperatures of the cascade regions returned to near the original crystal temperature of $10 \mathrm{~K}$, and no further defect motion was detectable on the time scale of MD simulations. The MD output for each cascade was analyzed to identify vacancies and SIAs, and then each cascade was read into ALSOME as a list of point defect locations. ALSOME immediately assigns each defect to a lattice site and performs defect interactions according to the critical reaction distances. Thus, the starting configuration consists of a distribution of defect clusters (of from $n=1, n_{\max }$ defects), each associated with a lattice site. 
The two cascades were each annealed 100 times for simulated annealing times of $1000 \mathrm{~s}$, and the numbers of each type of defect remaining were averaged. This was done for 17 temperatures between $0 \mathrm{~K}$ and $600 \mathrm{~K}$. The cascade region was assumed to rise instantaneously from $10 \mathrm{~K}$ to the annealing temperature at the beginning of each anneal.

A rectangular annealing volume was defined for each cascade that extends 25 lattice parameters beyond the maximum and minimum rectangular coordinates of the defect distribution. Any defect that goes outside this volume is assumed to have escaped from the cascade and is no longer followed. The annealing volume and time are consistent with cascade production in a typical fast reactor irradiation, i.e., on average another cascade will not occur in this volume during this time. These results are for isolated cascades only, and they do not reflect interactions of the defects with defects from prior or subsequent cascades in the material.

Figure 1 shows the total number of surviving defect pairs as a function of annealing temperature. These values include all defects that survive annihilation, including the escaping defects. Recovery Stages I, III and $V$ are evident at temperatures of approximately $0.03 \mathrm{~T}_{\mathrm{m}}, 0.15 \mathrm{~T}_{\mathrm{m}}$, and $0.32 \mathrm{~T}_{\mathrm{m}}$, respectively, as indicated by the decreases in the value of the total surviving defects. Escaping vacancies and escaping SIAs are also plotted separately in Fig. 1. At Stage I small SIA clusters and glissile loops become significantly mobile. The fraction of SIAs that escape the cascade is independent of temperature above that temperature. The SIAs lie at the 
periphery of the cascade region, and they will migrate more readily than vacancies at any temperature. The surviving SIAs remaining in the cascade region are in a few large clusters.

At temperatures in the somewhat broad Stage II, between $0.12-0.18 \mathrm{~T}_{\mathrm{m}}$, smal1 vacancy clusters become mobile, and nearly all escape the cascade region during the anneal, except for a few that annihilate at the stable, stationary large SIA clusters. Above Stage $V$, at $0.3 \mathrm{~T}_{\mathrm{m}}$, the vacancy clusters in the cascade core begin to dissociate, and nearly all of them also escape the cascade region. Thus, at temperatures of Stage $V$ and above, nearly twice as many vacancies as SIAs escape the cascades to become free defects. In this simulation, where SIA loops up to size $n=9$ were considered mobile, about half the escaping SIAs are in the form of glissile loops that move randomly in one dimension. The SIAs in glissile loops will move rapidly to sinks, compared to those in small SIA clusters, which make three-dimensional random walks. This differential in production of free vacancies relative to free SIAs for defects produced in cascades is the "production bias" that may be responsible for void swelling [2], which begins at temperatures above Stage V.

\section{ALSOME in the Multimodel Approach for High Energies}

The MMA defines a procedure for simulating defect production through the annealing stage for high energy cascades beyond the capabilities of MD. Cascades are generated through the collisional stage using the binary 
collision approximation ( $B C A)$, then a modified version of ALSOME is used to simulate the defect arrangement arrived at during quenching. The regular version of ALSOME is used for annealing the quenched cascades in the same way as was done for the MD cascades described in the previous example. The crucial step of the MMA is to devise a version of ALSOME that, after operating on the collisional phase "defect" distribution, yields a reasonable description of the actual defect distribution remaining after quenching. The term "defect" is applied loosely here because at the end of the collisional phase, no stable defects exist; there is only a collection of displaced atoms and vacant lattice sites. Results of MD simulations are used to calibrate the MMA quenching procedure, which is described in detail in Ref. [3].

In the initial attempts to devise a modified ALSOME for simulating the quenching operation for the BCA cascades, extensive use has been made of the two MD simulations of $25 \mathrm{keV}$ cascades in copper [8] described in the annealing simulation example above. To calibrate the quenching operation, collisional phase information was extracted from the two $25 \mathrm{keV}$ MD cascades in the same form as if it had been produced with the BCA code MARLOWE [9]. The ALSOME quenching scheme was then applied to the MARLOWE-like collisional stage configurations, and the ALSOME parameter values used during quenching were varied until the best agreement was obtained between the fully MD and MMA versions, as shown in Table 1. Of course, it is not possible to duplicate the actions of atoms or defects during the development and quenching of the thermal spike using a model in which defects simply move and interact. The criterion for agreement of the MMA and the fully MD versions of the cascades 
was taken to be similarity of the defect configurations following the annealing stage for annealing at $300 \mathrm{~K}$. In particular, the total number of defect pairs remaining after the quench and the total number of pairs and the numbers of free defects remaining after annealing could be made to agree for the MMA and the fully MD versions of the cascades, as shown in Table 1.

While the numbers of defects agree we11, the spatial distributions of the defects after quenching were significantly different for the MMA and MD versions. The distribution of SIAs had the same size (the root mean square average radius of the defects from the centroid) in both the MMA and MD versions, but the vacancy distribution after quenching in the MMA version was much broader than in the MD version. The vacancy distributions of the fully MD cascades after the quenching stage diminished to an average radius about half that of the initial collisional stage, whereas in the MMA case the average radius remained unchanged after quenching. Apparently, these differences in the spatial distributions do not affect the ability of this simple model to correctly partition the defects with respect to annihilation, clustering and free defects, at least at room temperature. Further attempts at this BCA-MD calibration using ALSOME will demand the temperature dependence of defect production after annealing to be consistent between the MMA and MD versions. This may require that an ALSOME quenching scheme be devised to produce the correct spatial distribution of defects in the quench, if possible. 


\section{Conclusions}

Two examples of ALSOME simulations are described here: the temperature dependence of differential defect production in cascades and the investigation of ALSOME as an approach to simulating quenching in high energy cascades. These examples illustrate the use of defect-scale Monte Carlo annealing simulations as the critical modeling link between the atomistic description of defect production and continuum theories of microstructure evolution under irradiation. Thus, in principal, the radiation damage process can be modeled at a level of physical reality relevant to the time and size scale at each stage from the initial atomic collisions to the development of microstructural features. 


\section{REFERENCES}

[1] T. Diaz de 1a Rubia and M.W. Guinan, J. Nucl. Mater. 174 (1990) 151.

[2] C.H. Woo and B.N. Singh, Phil Mag. A65 (1992) 889.

[3] H.L. Heinisch and B.N. Singh, J. Nucl, Mater. in press

[4] H.L. Heinisch, Rad. Eff. Def. Solids 113 (1990) 53.

[5] A.J.E. Foreman, W.J. Phythian and C.A. English, Phil. Mag. A 66 (1992) 671.

[6] H. Trinkaus, B.N. Singh and A.J.E. Foreman, J. Nucl. Mater. 206 (1993) 200.

[7] H.L. Heinisch and B.N. Singh, 17th ASTM Symposium on Effects of Radiation on Materials, June 20-23, 1994, Sun Valley, ID, to be published in $\mathrm{J}$. Nucl. Mater.

[8] T. Diaz de la Rubia and M.W. Guinan, Mater. Res. Forum 97-99 (1992) 23.

[9] M.T. Robinson and I.M. Torrens, Phys. Rev. B 9 (1974) 5008. 


\section{FIGURE CAPTION}

FIGURE 1. Annealing of Isolated $25 \mathrm{keV}$ Cascades in Copper. The defect fractions for the total number of defects surviving recombination and the vacancies and SIAs that escape from the cascade are plotted as a function of annealing temperature. Each data point is the average of 100 anneals of each of the two $25 \mathrm{keV}$ cascades generated in MD simulations [8]. The defect fractions are relative to the number of point defects existing after the quenching stage. The temperatures are relative to the experimental melting temperature of copper. 
TABLE 1

Defect Production in $25 \mathrm{keV}$ Cascades in $\mathrm{Cu}$ at $300 \mathrm{~K}$. MD cascades at the "quenching stage" (the end of the MD simulation) were only annealed with ALSOME. Those starting from the "collisional stage" (extracted from the MD simulations) were quenched using the ALSOME quenching calibration, then annealed with ALSOME.

\begin{tabular}{|l|c|c|c|c|}
\hline \hline & \multicolumn{2}{|c|}{ TOTAL DEFECT PAIRS } & \multicolumn{2}{c|}{ FREELY MIGRATING } \\
STARTING CONFIGURATION & QUENCHED & ANNEALED & VACANCIES & SIAS \\
\hline \hline QUENCHING STAGE & 60 & $49 \pm 4$ & $8 \pm 4$ & $9 \pm 3$ \\
\hline COLLISIONAL STAGE & $61 \pm 10$ & $48 \pm 7$ & $8 \pm 3$ & $9 \pm 4$ \\
\hline \hline
\end{tabular}




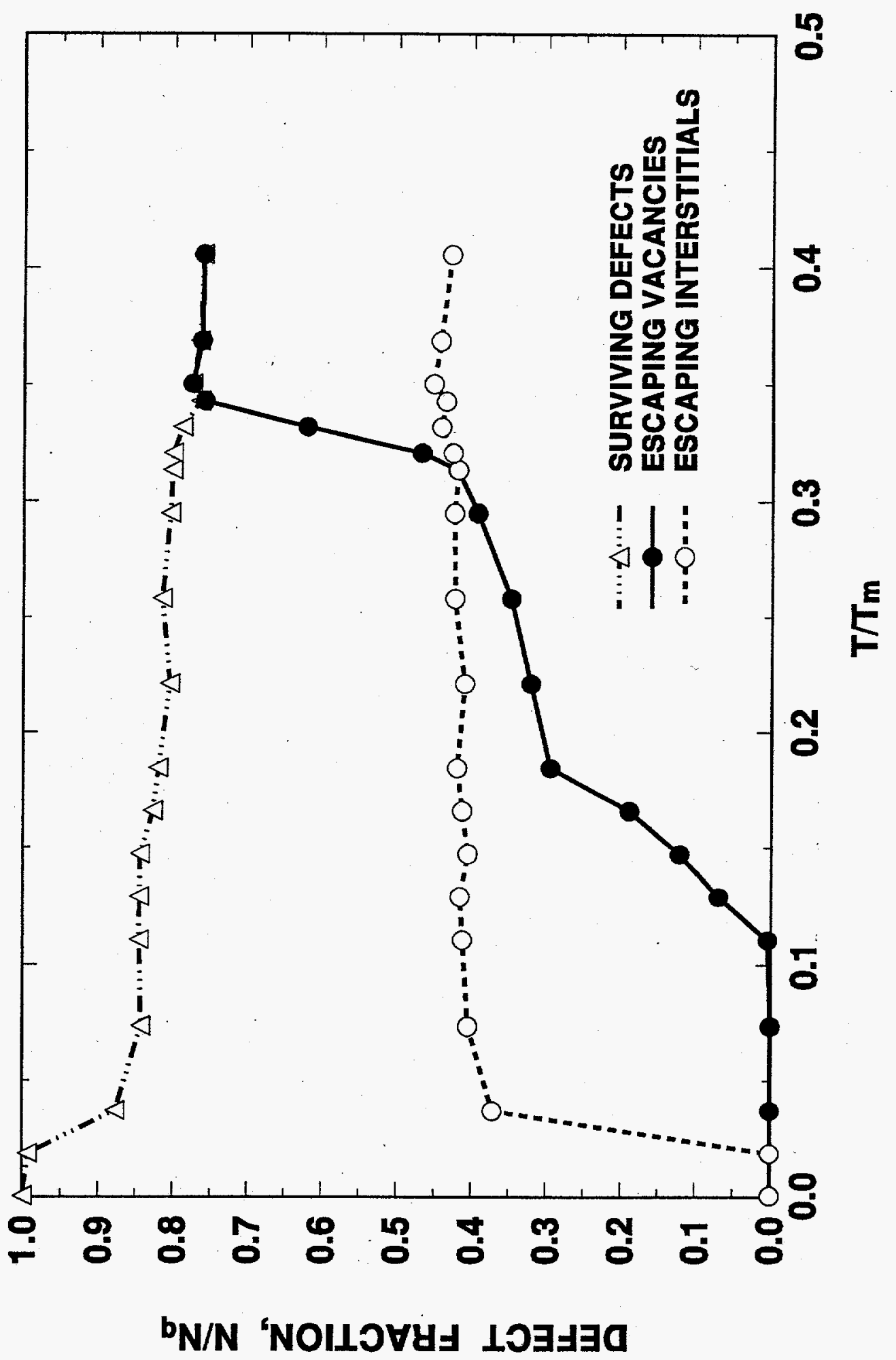

